\title{
CHEMICAL REACTIONS UNDER INCOMPLETE MIXING
}

\author{
OSATO MIYAWAKI ${ }^{1}$, HiRoo TSUJIKAWA \\ AND YUZO URAGUCHI \\ Department of Chemical Engineering, University of Tokyo, Tokyo, 113
}

\begin{abstract}
A degree of micro-mixing with a clear physical meaning is introduced to construct a universal reaction model which is applicable to reactions of any order. The model was applied to the multi-nozzle mixing system with two kinds of reaction system, $\mathrm{CO}_{2}-\mathrm{NH}_{3}$ and $\mathrm{CO}_{2}-\mathrm{NaOH}$, and was proved to explain the experimental results fairly well. The model was further applied to the batch tank reactor with alkaline hydrolysis of ethyl acetate. Also in this case, the trial was successful to some extent though some simplification was made during its course.
\end{abstract}

\section{Introduction}

The performance of a reactor with nonlinear reaction rate under incomplete micro-mixing cannot be predicted simply by the residence time distribution. Danckwerts $^{2)}$ first pointed out this fact and proposed complete segregation model (C. S. Model). His idea was extended by Zwietering ${ }^{18)}$ to propose a maximum mixedness model (M. M. Model). These studies established the concept of micromixing in contrast with macro-mixing. The latter is related to residence time distribution and the former is represented in terms of the intensity of segregation. The intensity of segregation, however, can explain the performance of a reactor only when C. S. Model or M. M. Model is applicable. These two models deal with the two limiting points in micro-mixing. At the intermediate state between them the intensity of segregation cannot describe the reactor performance completely. Weinstein and Adler ${ }^{16)}$ tried to solve this problem by a consecutive combination or a parallel combination of C.S. and M. M. Model. Recently Nishimura and Matsubara $^{18)}$ proposed a two-environmental model. This model covers the model of Weinstein and Adler as its one special case. The degree of micro-mixing is represented by segregation function instead of intensity of segregation here.

Compared with the above theoretical investigations, experimental studies in this field seem to be meager. With regard to stirred vessels, LaRosa and Manning ${ }^{7)}$ confirmed actual existence of segregation. Worrell

Received May 11, 1974.

Presented at the 6th Autumn Meeting of the Soc. of Chem. Engrs., Japan, Nov. '72.

1 Address for correspondence: Dept. of Agricultural Chem., Univ. of Tokyo, Tokyo, 113. and Eagleton ${ }^{17)}$ showed that C. S. Model is applicable at lower stirring rate and M. M. Model is applicable at higher rate. In a tubular reactor the problem discussed here mainly takes the form of turbulent mixing. Concerning this case there are investigations of Vassilatos and Toor ${ }^{15)}$, Kattan and Adler ${ }^{5)}$ and Harris and Stivastava ${ }^{3}$. In these three works, their respective models were constructed to be applied to the experimental results of Vassilatos and Toor ${ }^{15}$. Their attempts succeeded to some extent but their models don't necessarily have concrete physical meaning.

In this paper the degree of micro-mixing with a concrete physical meaning is first introduced and a universal mixing reaction model is established. This model is then applied to a multi-nozzle mixing system with two kinds of reaction and further applied to a stirred vessel.

\section{Theory}

Consider the case where two miscible solutions containing chemical species labeled 1 and 2, respectively, are being mixed by turbulence and 1 and 2 are simultaneously reacting with each other. The reaction occurring is assumed to be the following bimolecular $(m+n)$-th order reaction.

$$
\begin{aligned}
& \nu_{1} R_{1}+\nu_{2} R_{2} \rightarrow \text { product } \\
& r=-\left(\mathrm{d} C_{1} / \mathrm{d} t\right)=k C_{1}^{n} C_{2}^{n}
\end{aligned}
$$

Two solutions are divided into many lumps of small scale, these lumps are twisted and divided into much smaller scale by turbulence, and finally the solutions are mixed to molecular scale and then react with each other.

If one of these lumps being subjected to mixing is taken out at one point it may have a structure as shown in Fig. 1, where sections $A, B$ are molecular-mixed 


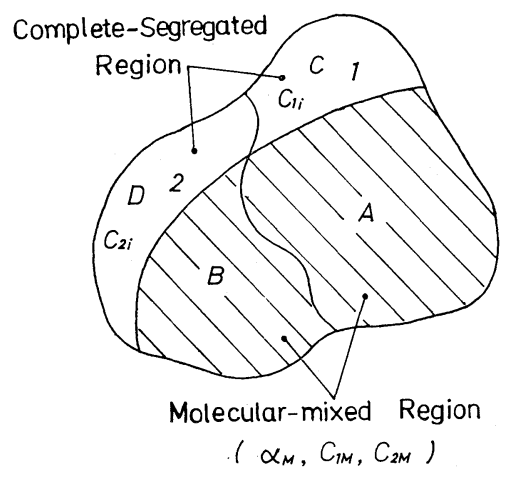

Fig. 1 Structural model of a liquid lump

regions and $C, D$ are complete segregated regions. The relative volume ratio of solution 1 and 2 is assumed to be the same as the total average of the mixing system. These assumptions may simplify the phenomena too much, and in the more exact analysis a statistical approach such as assuming the probability distribution about the volume ratio of 1 must be considered. However, as a first approximation, we can take the assumption above. Here the degree of micro-mixing $\alpha_{M}$ is defined as relative volume ratio of the summation of the molecular-mixed region to the total mixing system.

In a tubular mixer $\alpha_{M}$ is assumed to have a constant value in the plane perpendicular to the axial direction and changes with this direction. In an isotropic stirred vessel, $\alpha_{M}$ is assumed to have a constant value throughout the vessel and changes with time if it is operated unsteadily.

With these assumptions the following equations are derived.

$$
\begin{aligned}
& A+C=\bar{\alpha} \\
& B+D=1-\bar{\alpha} \\
& A / B=\bar{\alpha} /(1-\bar{\alpha}) \\
& A+B=\alpha_{M} \\
& C_{1 i} C+C_{1 M} \alpha_{M}=\bar{C}_{1} \\
& C_{2 i} D+C_{2 M} \alpha_{M}=\bar{C}_{2}
\end{aligned}
$$

where $\bar{C}_{1}$ and $\bar{C}_{2}$ are the average concentrations of component 1 and 2 respectively and $\bar{C}_{10}$ and $\bar{C}_{20}$ are its initial values. $C_{1 i}$ and $C_{2 i}$ are the initial nonaveraged values. $C_{1 M}$ and $C_{2 M}$ are the average concentrations of 1 and 2 in the molecular-mixed region.

With these equations, $C_{1 M}$ and $C_{2 M}$ are calculated as follows.

$$
\begin{aligned}
& C_{1 M}=\left[\bar{C}_{1}-\bar{C}_{10}\left(1-\alpha_{M}\right)\right] / \alpha_{M} \\
& C_{2 M}=\left(\nu_{2} / \nu_{1}\right)\left[\bar{C}_{1}-\bar{C}_{10}\left(1-\beta \alpha_{M}\right)\right] / \alpha_{M}
\end{aligned}
$$

where $\beta$ is the excess feed ratio for reactant 2 , i.e.

$$
\beta=\nu_{1} \bar{C}_{20} / \nu_{2} \bar{C}_{10}
$$

The reaction is assumed to occur only in the molecular-mixed region. Then apparant rate of the reaction is expressed as follows.

$$
-\left(\mathrm{d} \bar{C}_{1} / \mathrm{d} t\right)=k C_{1 M}^{m} C_{2 M}^{n} \alpha_{M}
$$

Eqs. (9) and (10) are substituted into Eq. (12) and the fractional conversion, $F\left(=1-\bar{C}_{1} / \bar{C}_{10}\right)$ is introduced. Then Eq. (12) becomes

$$
\begin{aligned}
\left(1 / \alpha_{M}\right)(\mathrm{d} F / \mathrm{d} t)= & k\left(\nu_{2} / \nu_{1}\right)^{n} C_{10}^{m+n-1}\left[1-\left(F / \alpha_{M}\right)\right]^{m} \\
& \times\left[\beta-\left(F / \alpha_{M}\right)\right]^{n}
\end{aligned}
$$

This means that the reaction under incomplete mixing can be described with an apparent change of conversion $\left(1 / \alpha_{M}\right)(\mathrm{d} F / \mathrm{d} t)$ and an apparent conversion $\left(F / \alpha_{M}\right)$ instead of $(\mathrm{d} F / \mathrm{d} t)$ and $F$, respectively, in the case of a homogeneous reaction system. Eq. (13) converges into a homogeneous equation when micromixing is complete, i.e. $\alpha_{M} \rightarrow 1$. In the case of secondorder reaction with $m=n=1$, Eq. (13) becomes

$$
\left(1 / \alpha_{M}\right)(\mathrm{d} F / \mathrm{d} t)=\left(k \nu_{2} \bar{C}_{10} / \nu_{1}\right)\left[1-\left(F / \alpha_{M}\right)\right]\left[\beta-\left(F / \alpha_{M}\right)\right]
$$

The next problem concerns the quantity to be used as $\alpha_{M}$. There seem to be two ideas concerning this problem. According to Toor's theory ${ }^{13)}$ fractional conversion $F_{R}$ of a very rapid reaction between two miscible fluids under turbulent mixing is related to fractional completion of mixing $\eta$.

$$
\begin{gathered}
1-F_{R}=\eta=\sqrt{\overline{c^{\prime 2}}} /\left(\sqrt{c^{\prime 2}}\right)_{\mathrm{ref}} \quad \text { at } \beta=1 \\
1-F_{R}=-[(1-\beta) / 2](\gamma \eta) \text { ierfc }(1 / \gamma \eta)=f(\beta, \eta) \\
\text { at } \beta \neq 1
\end{gathered}
$$

where $\left(\sqrt{c^{\prime 2}}\right)_{\text {ref }}$ is a correction factor to relate $F_{R}$ to $\sqrt{c^{\prime 2}} . \quad \gamma$ is a function of $\beta$ and is numerically determined from the initial value of $F_{R}$, therefore $F_{R}$ is a function of $\beta$ and $\eta$.

$F_{R}$ or $1-\eta$ seems to be suitable for our purpose, i.e.

$$
\alpha_{M}=F_{R}
$$

or

$$
\alpha_{M}=1-\eta
$$

Both Eqs. (17) and (18) take the value of zero in the state of complete segregation and unity in the state of complete molecular mixing. So they seem to satisfy the fundamental conditions to be adopted as $\alpha_{M}$. $F_{R}$ is dependent on $\beta$ but $(1-\eta)$ is not. At $\beta=1$ they coincide with each other and at $\beta \neq 1$ they are related to each other through Eq. (16), thus the two kinds of $\alpha_{M}$ can be calculated from the information of either $F_{R}$ or $\sqrt{\overline{c^{\prime 2}}}$. Comparison of Eqs. (17) and (18) will be discussed later. With Eq. (17) or (18), apparant behavior of a reaction under incomplete mixing can generally be described by Eq. (13).

In the case of $\beta=1, \alpha_{M}$ was selected appropriately and Eq. (14) was numerically integrated with various values of rate constant. Results are shown in Fig. 2. The following will be easily understood from the figure. The effect of mixing increases with the in- 
increase of rate constant $k$; furthermore, nonhomogeneous results converge to that for the case of very rapid reaction of $\left(k \nu_{2} C_{10} / \nu_{1}\right) \rightarrow \infty$.

\section{Experimental}

\section{1. Multi-nozzle tubular mixer}

$40^{\phi}$ Lucite pipe was used as a mixer, whose inlet held 24 nozzles. The diagram of the flow system was presented in the author's preceeding paper $^{8}$. The reaction sysems selected are $\mathrm{CO}_{2}-\mathrm{NH}_{3}$ and $\mathrm{CO}_{2}-$ $\mathrm{NaOH}$ in aqueous solution. The solution of $\mathrm{CO}_{2}$ was prepared by adding dry ice to the water in a reservoir. The solutions of $\mathrm{NH}_{3}$ and $\mathrm{NaOH}$ were also prepared in a reservoir. In the case of $\mathrm{CO}_{2}-\mathrm{NH}_{3}$, $\mathrm{NH}_{4} \mathrm{Cl}$ was added to the solution of $\mathrm{NH}_{3}$ to suppress the conversion of $\mathrm{NH}_{3}$ to $\mathrm{NH}_{4}^{+}$. The same quantity of $\mathrm{NH}_{4} \mathrm{Cl}$ was added also to the $\mathrm{CO}_{2}$ solution to prevent the effect of heat of dilution.

Fractional conversion was determined from measurement by a thermocouple of temperature rise of the solution due to the reaction. Therefore the two solutions to be mixed were adjusted to the same temperature. Initial concentation was measured by titration. In each operation alkaline solution was supplied in excess to complete the reaction. The experimental results were analyzed on the assumption of perfect completion of the reaction at the outlet of the mixer, which was $1000 \mathrm{~mm}$ long.

\section{2. Stirred vessel}

A Rushton-type stirred vessel with six-blade turbine, shown in Fig. 3, was used. It was set in a constanttemperature water bath. Batch-wise mixing and reaction was performed in it. The very rapid reaction between $\mathrm{HCl}$ and $\mathrm{NaOH}$ was used as the tracer of mixing to measure the mixing time. Hydrolysis of $\mathrm{CH}_{3} \mathrm{COOC}_{2} \mathrm{H}_{5}$ by $\mathrm{NaOH}$ was selected as the reaction system under incomplete mixing.

In each of the two the progress of the reaction was pursued by the electric conductivity method. The linearity of the electric conductivity and the concentration of electrolyte were checked prior to the experiment. PVA was added to the solution to increase its viscosity. The procedure to start the reaction was as follows. $2500 \mathrm{ml} \mathrm{NaOH}$ solution was poured into the stirred vessel in the water bath and made to attain temperature equilibrium. Then the counterpart solution of about 1/10 the volume was injected promptly into the vessel so that the reaction was started. Injecting point of the second solution and position of the conductivity probe are shown in Fig. 3.

\section{Results and Discussion}

\section{1. Multi-nozzle tubular mixer}

The reaction rate of $\mathrm{CO}_{2}-\mathrm{NH}_{3}$ system is of second-

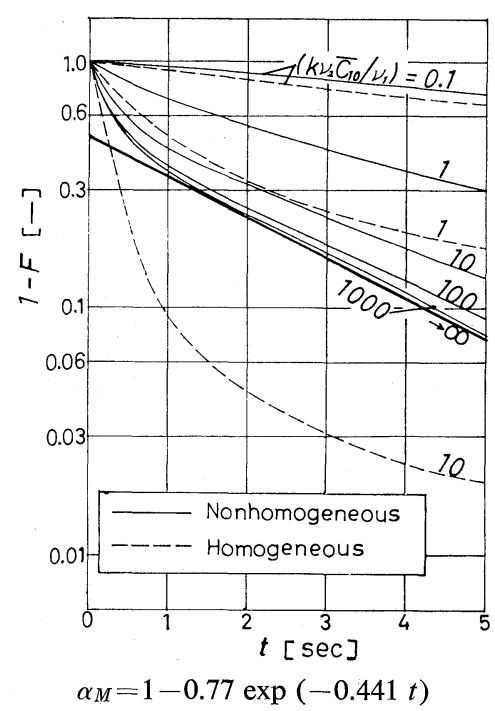

Fig. 2 Numerical integration of Eq. (14) at $\beta=1$

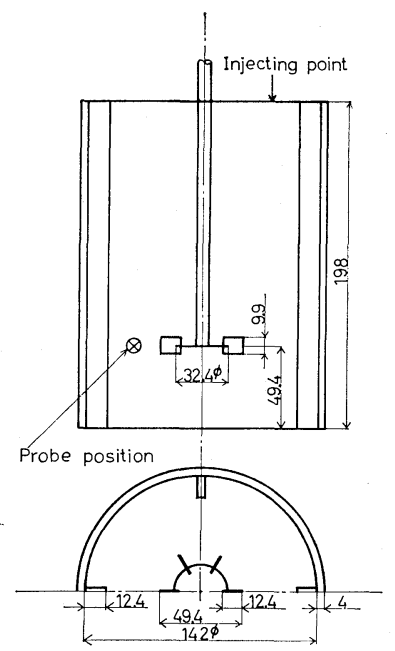

Fig. 3 Stirred vessel and turbine

order and the rate constant is given by Pinsent et al. ${ }^{12)}$.

$$
\begin{aligned}
& -\frac{\mathrm{d}\left[\mathrm{CO}_{2}\right]}{\mathrm{d} t}=k\left[\mathrm{CO}_{2}\right]\left[\mathrm{NH}_{3}\right] \\
& k=10^{11.13} \exp (-11600 / R T)
\end{aligned}
$$

In this case mixing is faster than reaction.

The reaction of $\mathrm{CO}_{2}-\mathrm{NaOH}$ was also studied by Pinsent et $a l^{11}$.

$$
\begin{aligned}
& -\frac{\mathrm{d}\left[\mathrm{CO}_{2}\right]}{\mathrm{d} t}=k\left[\mathrm{CO}_{2}\right]\left[\mathrm{OH}^{-}\right] \\
& k=4.2 \times 10^{13} \exp (-13250 / R T)
\end{aligned}
$$

In this case, mixing is slower than reaction as opposed to the case for the former system.

Information concerning rate of mixing is necessary in the next place. The mixing characteristics of multi-nozzle mixer were presented in the preceding paper $^{8)}$, where the time constant $t_{M}$ corresponding to the decay of $c^{\overline{2}}$ was expressed by four parameters $\bar{U}, d_{N}, \bar{\alpha}$ and $S c$, namely,

$$
t_{M}=(1 / 2)\left\{3 / \varepsilon^{1 / 3} k_{0 c}^{2 / 3}+(\nu / \varepsilon)^{1 / 2} \log (S c)\right\}
$$




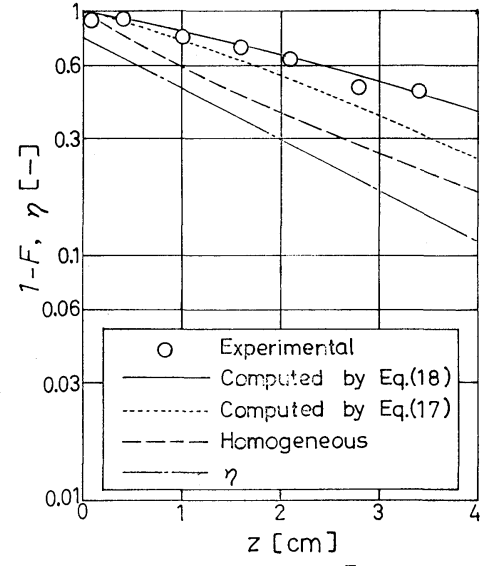

$\left(\theta=17.7^{\circ} \mathrm{C}, \quad \bar{U}=15.9 \mathrm{~cm} / \mathrm{sec}, \bar{C}_{10}=0.0316 \mathrm{eq} / l\right.$, $\beta=2.19 \mathrm{eq} / \mathrm{eq}, k=262 \mathrm{l} / \mathrm{mol} \cdot \mathrm{sec}$ )

Fig. $4 \mathrm{CO}_{2}-\mathrm{NH}_{3}$ in multi-nozzle tubular mixer

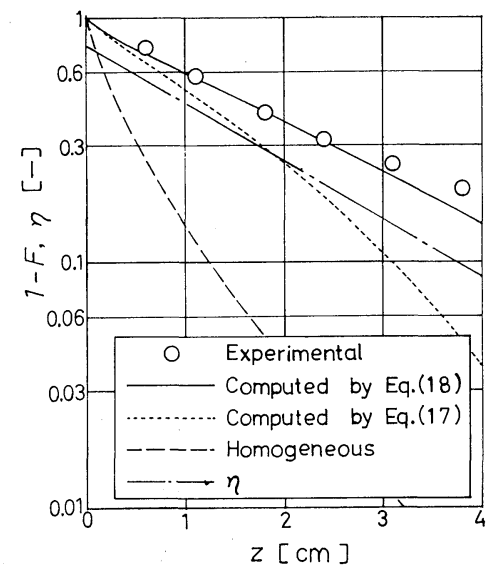

$\left(\theta=17.3^{\circ} \mathrm{C}, \bar{U}=39.7 \mathrm{~cm} / \mathrm{sec}, \bar{C}_{10}=0.0390 \mathrm{eq} / l\right.$, $\beta=1.51 \mathrm{eq} / \mathrm{eq}, k=4598 \mathrm{l} / \mathrm{mol} \cdot \mathrm{sec})$

Fig. $5 \mathrm{CO}_{2}-\mathrm{NaOH}$ in multi-nozzle tubular mixer

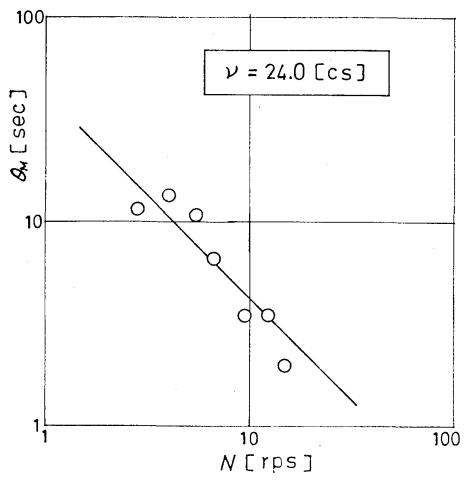

Fig. 6 Relation between $95 \%$ mixing time and rotational speed

$$
\varepsilon=\left\{0.133 \bar{U} \sqrt{\left(2 \bar{\alpha}^{2}-2 \bar{\alpha}+1\right) / 0.75 d_{N}^{2}-1}\right\}^{3}\left(2 \pi / d_{N}\right)
$$

$$
k_{0 c}=12.5
$$

Using these equations and Eq. (15), fractional completion of mixing $\eta$ is expressed as

$$
\eta=0.77 \exp \left(-t / 2 t_{M}\right)
$$

where a coefficieint 0.77 is the typical value for all the data in the preceding paper. With Eqs. (19) (26) conversion can be calculated from Eq. (14). In Figs. 4 and $5,1-F$ of experimental, one calculated based on the two kinds of $\alpha_{M}$ and one calculated with an assumption of homogeneous mixing, are shown along with $\eta$. As can be seen from these figures, the calculated results using $\alpha_{M}$ from Eq. (18) coincide with the experimental ones fairly well. This is true throughout all 20 data for $\mathrm{CO}_{2}-\mathrm{NH}_{3}$ system and 10 data for $\mathrm{CO}_{2}-\mathrm{NaOH}$. The disagreement of the computational results based on $F_{R}$ is rather unexpected. Eq. (13) converges to $F=\alpha_{M}$ when $k \rightarrow \infty$. Therefore, if Toor's theory is sound, $\alpha_{M}$ must be equal to $F_{R}$ when $k$ becomes large. The disagreement may suggest the insufficiency of his theory. The estimation of $F_{R}$ from Eq. (16) has a tendency to overestimate the experimental values a little ${ }^{6}$.

Here comparison is made with other models. Vassilatos and Toor ${ }^{15)}$ investigated second-order reactions under incomplete mixing. Assuming the rate of mixing as second order, they evaluated apparent second-order rate constant $K$ as

$$
(1 / K)=\left(1 / k_{r}\right)+\left(1 / k_{m}\right)
$$

where $k_{r}$ and $k_{m}$ are the rate constant of reaction and mixing, respectively. But in their model there may be some unreasonableness in assuming the rate of mixing as second order and, perhaps as a result, the model couldn't explain the experimental results for larger $k_{r}$.

Kattan and Adler $^{5)}$ and Harris and Srivastava ${ }^{3)}$ proposed independently a stochastic model where the fluid under mixing is divided into many cells, and a random instantaneous coalescence and redispersion step among the cells and a homogeneous reaction step in each cell are repeated in turn. This model could explain the experimental results of Vassilatos and Toor $^{15)}$. The model, however, includes many parameters and there must be some arbitrariness in determining them. It is a model for the computer and so does not have such a clear meaning as Eq. (13). Moreover, the model requires considerably long time for computation. These seem to be weak points of the model.

\section{2. Stirred vessel}

In the case of a stirred vessel there is no information on the degree of micro-mixing $\alpha_{M}$. To determine it, the mixing time $\theta_{M}$, the duration from the start of the mixing to $95 \%$ completion of the mixing, was measured first. The very rapid reaction of $\mathrm{HCl}$ and $\mathrm{NaOH}$ was used as a tracer reaction and the decrease of electroconductivity with the progress of mixing was traced. Thus obtained $\theta_{M}$ was plotted against the rotational speed of the turbine $N$ in Fig. 6 . The relation between $\theta_{M}$ and $N$ was as follows. 


$$
N \theta_{M}=\text { const }=42.3
$$

This inverse proportionality coincides with previous results ${ }^{4,9)}$.

$\alpha_{M}$ can be related to $\theta_{M}$. Through the measurement of $\theta_{M}$, mixing was found to progress exponentially on an average. Then $\alpha_{M}$ is expressed as follows.

$$
\alpha_{M}=1-\exp \left(-t / t_{M}\right)
$$

The time constant $t_{M}$ in Eq. (29) can be directly determined from $\theta_{M}$, because $\theta_{M}$ corresponds to the time for $\alpha_{M}=0.95$ and was calculated as

$$
t_{M}=14.1 / N
$$

In the next place, information on chemical kinetics is necessary. Concerning the $\mathrm{CH}_{3} \mathrm{COOC}_{2} \mathrm{H}_{5}-\mathrm{OH}^{-}$ system, the second-oder rate constant for lower temperature than used here is presented by Tsujikawa and Inoue ${ }^{14}$. In the present case, the solutions contain PVA, which seemed to hinder the reaction a little. This problem seems due to the $-\mathrm{OH}$ and $-\mathrm{COONa}$ groups of PVA. The relative amount of -COONa group in PVA was estimated as about $1 \%$ in equivalent. The effects of $-\mathrm{OH}$ and $-\mathrm{COONa}$ groups were qualitatively tested by adding methanol, ethanol and sodium acetate, respectively, into the solution without PVA. This type of prevention of reaction seems to be inevitable because all of the agents such as PVA used to increase viscosity contain $-\mathrm{OH}$ group or $-\mathrm{COONa}$ group. As a matter of fact the same reaction using CMC was tested and the rate constant obtained was proved to be considerably smaller than expected.

Because of the reasons mentioned above, the rate constant from the literature overestimate the experimental value by $20 \sim 30 \%$. So it was intended to determine the rate constant from the experiment free from the rate of mixing. However, such an experiment was again prohibited by the high viscosity of the PVA solution. Yet, under the effect of mixing, the order of the reaction was found to be second after the lapse of $\theta_{M}$ from the start, and the rate constant was obtained from the information at this period. This treatment may be acceptable because, in spite of the high viscosity of the solution, the rate of mixing is still much faster than the reaction rate. With this rate constant and $\alpha_{M}$ from Eqs. (29) and (30), conversion at the period from the start to time $\theta_{M}$ was calculated from Eq. (14).

The results obtained are shown in Fig. 7. Eq. (14) could explain the experimental results to some extent, but the agreement of the model and the experiment is not so good compared with the multinozzle case. There seem to be two reasons for this. First, the reaction was started by quick injection of the second reactant, so the initial condition was not

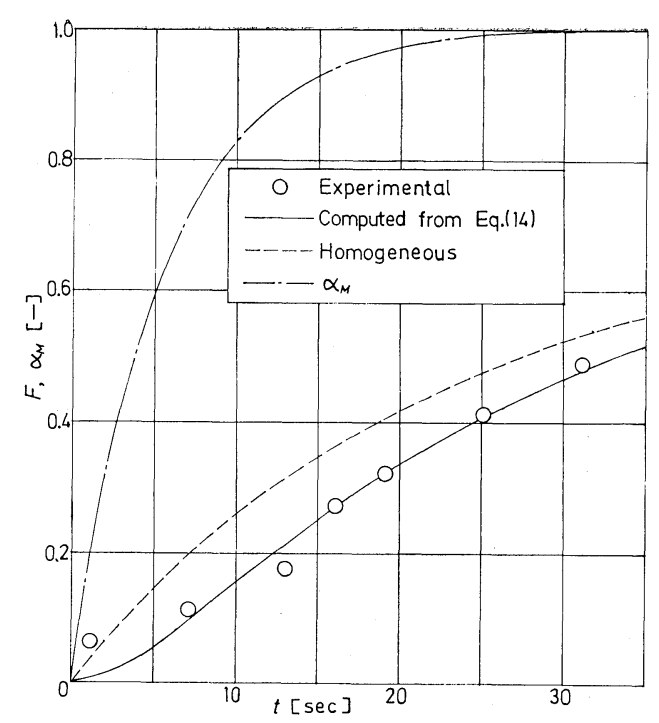

$\left(\theta=64.0^{\circ} \mathrm{C}, \bar{C}_{10}=0.0316 \mathrm{eq} / \mathrm{l}, \quad \beta=1.19 \mathrm{eq} / \mathrm{eq}, \quad k=0.830 \mathrm{l}\right.$ $\mathrm{mol} \cdot \mathrm{sec}, N=2.70 \mathrm{rps}, \nu=22.1 \mathrm{cs})$

Fig. 7 Alkaline hydrolysis of ethyl acetate in stirred vessel

necessarily fixed. Second, the model with one parameter $\alpha_{M}$ concerning mixing may be too rough for such reactors as a stirred vessel.

This model may be extended to the case of recycle flow in the vessel, because the degree of micro-mixing described here has a superimposing character. This model is expected to be applicable also to a continuous stirred vessel.

\section{Conclusions}

1. The degree of micro-mixing was defined as the relative volume ratio of the summation of the molecular mixed region to the total reaction system. A universal reaction model under incomplete mixing was constructed based on it. It took a very simple form and converged into the homogeneous equation when micro-mixing was complete.

2. The reactions of $\mathrm{CO}_{2}-\mathrm{NH}_{3}$ and $\mathrm{CO}_{2}-\mathrm{NaOH}$, respectively, in the multi-nozzle mixer were explained fairly well by the model based on $1-\eta$. In the former reaction mixing was faster than reaction and in the latter case the reverse was true. Comparison with other models was made.

3. The model was applied to alkaline hydrolysis of ethyl acetate in a stirred vessel. $\alpha_{M}$ was related to the mixing time in this case. Experimental results were explained by the model to some extent.

\section{Nomenclature}

$A, B, C, D=$ volume ratio

$a, b=$ stoichiometric coefficient

$\bar{C}_{1}, \bar{C}_{2}=$ averaged concentration

$[-]$

$\bar{C}_{10}, \bar{C}_{20}=$ averaged initial concentration

$C_{1 i}, C_{2 i}=$ nonaveraged initial concentration 


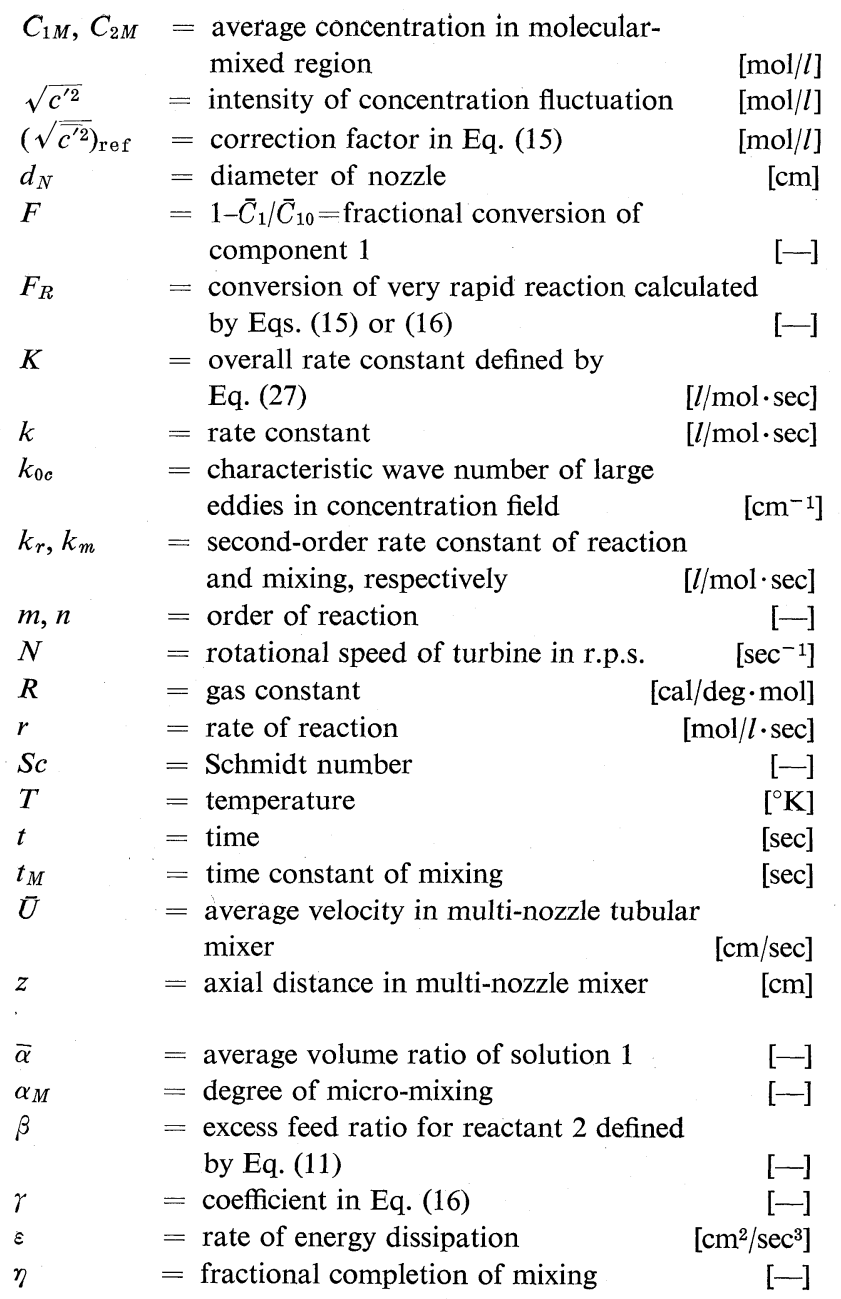

$\begin{array}{llr}\theta & =\text { temperature } & {\left[{ }^{\circ} \mathrm{C}\right]} \\ \theta_{M} & =\text { mixing time in stirred vessel } & {[\mathrm{sec}]} \\ \nu & =\text { kinetic viscosity } & {\left[\mathrm{cm}^{2} / \mathrm{sec}\right]}\end{array}$

\section{Literature Cited}

1) Brinkman, R., R. Margaria and F. J. W. Roughton: Phil. Trans. Roy. Soc., A, 232, 65 (1933).

2) Danckwerts, P. V.: Chem. Eng. Sci., 8, 93 (1958).

3) Harris, I. J. and R. D. Srivastava: Can. J. Chem. Eng., 46, 66 (1968).

4) Kamiwano, M., K. Yamamoto and S. Nagata: Kagaku Kōgaku, 31, 365 (1967).

5) Kattan, A. and R. J. Adler: AIChE J., 13, 580 (1967).

6) Keeler, R. N., E. E. Petersen and J. M. Prausnitz: AIChE J., 11, 221 (1965).

7) LaRosa, P. and F. S. Manning: Can. I. Chem. Eng., April, 65 (1964).

8) Miyawaki, O., H. Tsujikawa and Y. Uraguchi: J. Chem. Eng. Japan, 7, 52 (1974).

9) Nagata, S., M. Yanagimoto and T. Yokoyama: Kagaku Kögaku, 21, 278 (1957).

10) Nishimura, Y. and M. Matsubara: Chem. Eng. Sci., 25, 1785 (1970).

11) Pinsent, B. R. W., L. Pearson and F. J. W. Roughton: Trans. Faraday Soc., 52, 1512 (1956).

12) idem: ibid., 52, 1594 (1956).

13) Toor, H. L.: AIChE J., 8, 70 (1962).

14) Tsujikawa, H. and H. Inoue: Bull. Chem. Soc. Japan, 39, 1837 (1966).

15) Vassilatos, G. and H. L. Toor: AIChE J., 11, 666 (1965).

16) Weinstein, H. and R. J. Adler: Chem. Eng. Sci., 22, 65 (1967).

17) Worrell, G. R. and I. C. Eagleton: Can. J. Chem. Eng., December, 254 (1964).

18) Zwietering, Th. N.: Chem. Eng. Sci., 11, 1 (1959). 